\title{
TIMING SYNCHRONIZATION FOR MC-CDMA SYSTEMS USING A TIME-MULTIPLEXED SYNCHRONIZATION CHANNEL
}

\author{
Pham Hong $\mathrm{Ky}^{*}$ and Nguyen Duc Long \\ Research Institute of Post and Telecom \\ 122 Hoang Quoc Viet, Cau Giay, Hanoi, Vietnam
}

Received 25 November 2005

\begin{abstract}
In this paper, we present a synchronization algorithm for Multi-Carrier CDMA (MC-CDMA) systems using a time-multiplexed synchronization channel. The simulations results show that a system using proposed algorithm has much higher timing detection probability than using other algorithms, especially in exponential decay multipath fading.
\end{abstract}

\section{INTRODUCTION}

MC-CDMA (Multi Carrier Code Division Multiple Access) is one of principle multiple access methods proposed for next generation mobile communication system. However, like other multi-carrier systems, MC-CDMA system is limited by synchronization errors more than one carrier system. Synchronization errors reduce received signals strength and make its phase rotated, and cause ICI (Inter Carrier Interference) also. Due to ICI, the orthogonality between sub-carriers will be broken. This causes the performance reduction of the whole system.

In MC-CDMA systems, the signals can be spread in frequency or time domain by spreading code [1 - 3]. The synchronization is acquired by using information on the pilot and synchronization $(\mathrm{SCH})$ channel. $\mathrm{SCH}$ channel structure is shown in Fig. 1. There are some algorithms proposed to achieve the timing synchronization [4]: Detecting the correlation between received signals before FFT (Fast Fourier Transform) processing and SCH signals at the receiver, detecting the correlation between two consecutive received $\mathrm{SCH}$ in the timemultiplexed structure and detecting the Guard Interval correlation in frequency-multiplexed $\mathrm{SCH}$.

We will review the above-mentioned algorithms [4] along with their advantages, disadvantages and in the next section; we propose one new algorithm to improve the performance of the whole system.

We first present the correlation detection algorithm between the received signals before FFT processing and SCH signals at the receiver. In this algorithm, the SCH with one- or two-symbol duration is time-multiplexed into the coded data symbols at each sub-carrier at the beginning of each frame. The synchronization at the receiver is carried out by using one SCH-replica generated by performing IFFT (Inverse FFT) on the SCH. After calculating the correlation between the SCH-replica and the received signal sequence before FFT, the receiver averages the correlation values in squared form over several frame interval in order to decrease the impact of

${ }^{*}$ Corresponding author e-mail: phamhongky@hn.vnn.vn 
the interference and noise. Then, it finds the highest averaged correlation value and defines the timing.

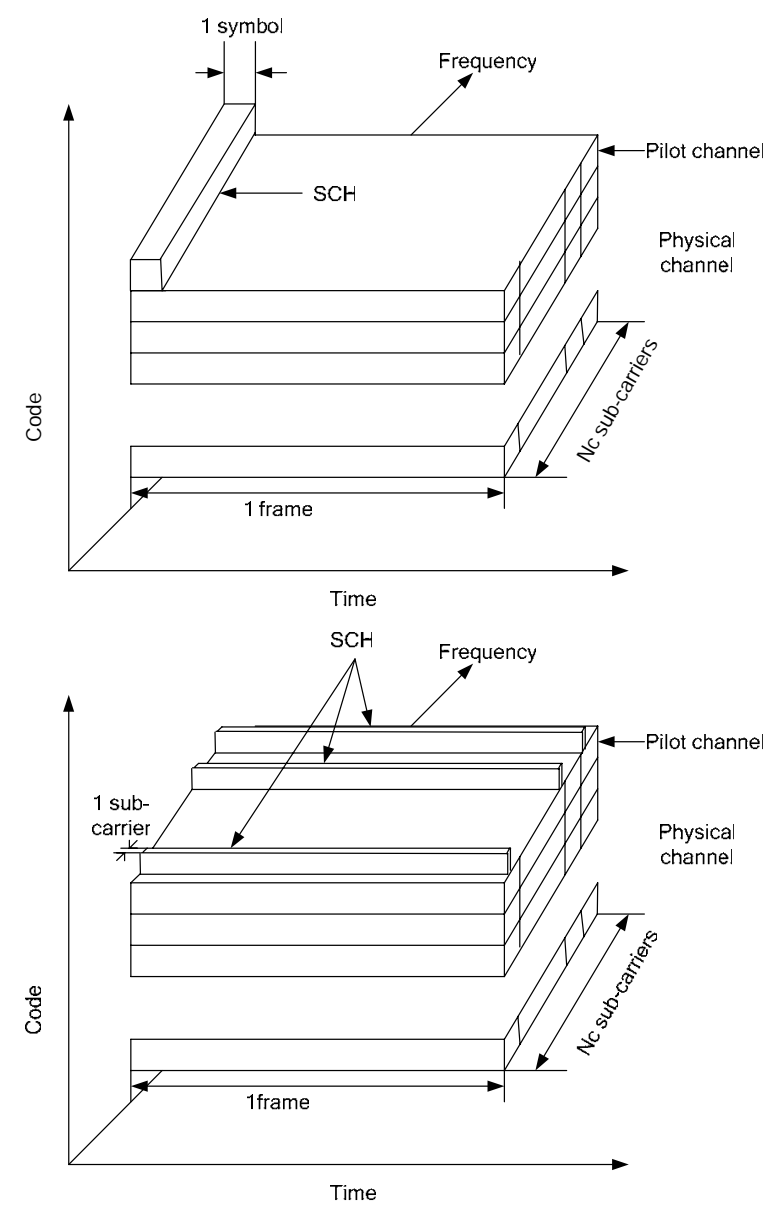

Fig. 1: Time-multiplexed SCH (above) and frequency-multiplexed SCH (below)

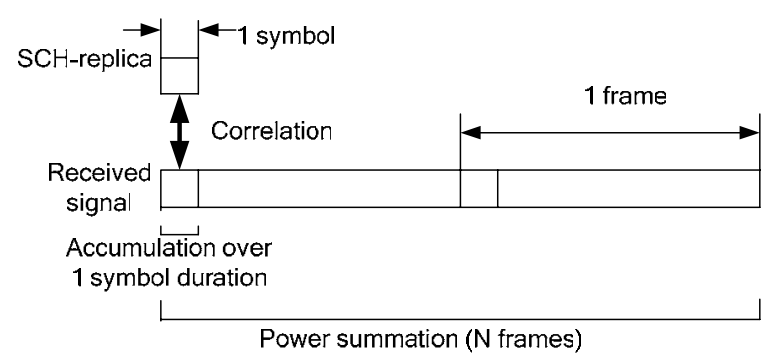

Fig. 2: Detecting the correlation between received signals before FFT processing and SCH signals at the receiver

The second algorithm is time-multiplexed synchronization with one-symbol-differentiated correlation method. This algorithm uses two successive $\mathrm{SCH}$ symbols multiplexed at the beginning of each frame. At the receiver, the correlation between the received signal and onesymbol delayed version of the signal per FFT sampling duration is calculated. After coherently 
accumulating the correlation values over several frames, searching the highest correlation output, the receiver can detect the FFT window and frame timing.

In two time-multiplexed $\mathrm{SCH}$ methods mentioned above, the results in [4] show that the algorithm using SCH-replica has a better performance and is widely used. However, its disadvantage is that the receiver must know the structure of the transmitted $\mathrm{SCH}$. Beside these two methods, the synchronization can be achieved by using the Guard Interval in the frequencymultiplexed SCH. Here, the SCH is frequency-multiplexed into the sub-carriers every $n$ subcarriers. The synchronization is taken into two steps: FFT window timing and frame timing.

FFT window timing is detected by using guard interval. The detector calculates the correlation between the received sequence and the version of that delayed by FFT window interval, then accumulates over all symbols of one frame. The timing is detected by searching the maximum value.

Frame timing is detected by using the frequency-multiplexed $\mathrm{SCH}$. Assuming that $\mathrm{SCH}$ is multiplexed into $k$ sub-carriers, the receiver can use the FFT window timing detected from the first step for FFT processing. Firstly, it takes the correlation between SCH-replica and FFT output of each sub-carrier in which the $\mathrm{SCH}$ is transmitted, then takes the sum of $k$-calculated correlations. Finally, taking the average over several frames and finding the maximum value.

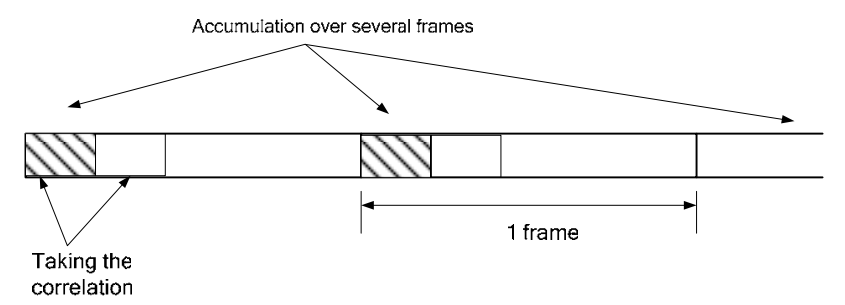

Fig. 3: Detecting the correlation between two received consecutive time-multiplexed SCH symbols

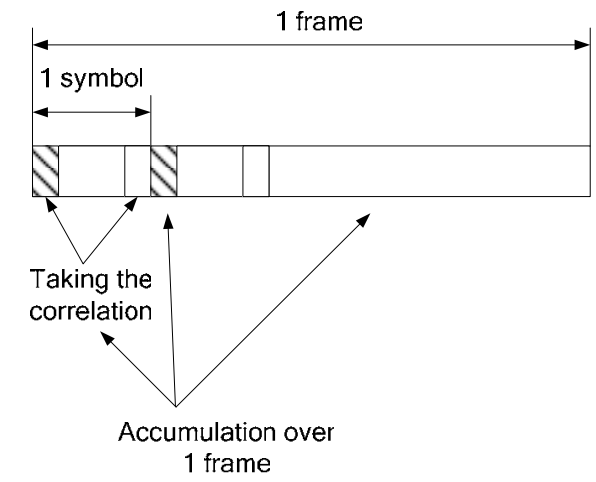

Fig 4: FFT window timing in frequency-multiplexed synchronization

Among these three algorithms, the algorithm that detects the correlation between two consecutive received time-multiplexed SCH symbols has the advantages of simplicity and the structure of transmitted $\mathrm{SCH}$ is not required at the receiver. However, its disadvantage is the low synchronization performance in multi-path fading channel [5]. In the next section, we will present this algorithm in detail and propose one modification to improve the performance. 


\section{THE SYNCHRONIZATION USING TWO CONSECUTIVE TIME-MULTIPLEXED SCH SYMBOLS AT THE BEGINNING OF EACH FRAME}

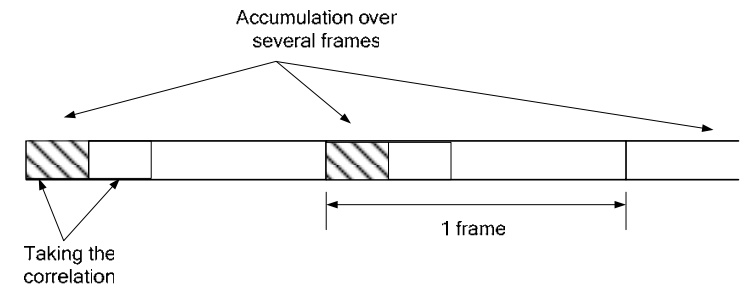

Fig. 5: Synchronization using 2 consecutive time-multiplexed SCH symbols at the beginning of each frame

In this synchronization method [4], the frame and the FFT window timing are found by searching the maximum value of the correlation between two consecutive symbols

$$
\Theta(k)=\sum_{1 \text { symbol }} r^{*}(k) r(k+N+G I)
$$

where $N$ is the FFT window length.

The disadvantage of this algorithm is that the plateau occurs in the correlation window, as shown in Fig. 6, so that it is difficult to define the peak. As consequence, the timing is not correct.

In order to eliminate the plateau, in [6], two symmetrical SCH symbols, as shown in Fig. 7, are used instead of the two identical ones .

The correlation is calculated as follow:

$$
\Theta(k)=\sum_{i=1}^{N} r^{*}(k-i) r(k+i)
$$

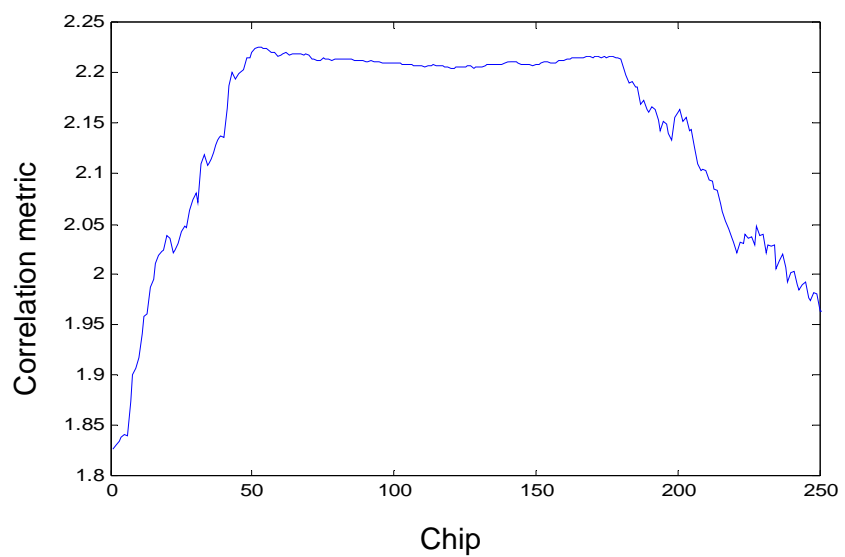

Fig. 6: Plateau in the timing metric 


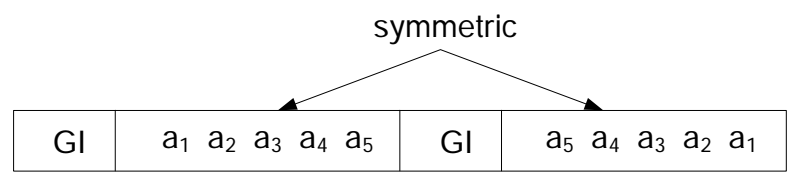

Fig. 7: SCH structure in modified algorithm

The correlation will reach the maximum value at correct timing.

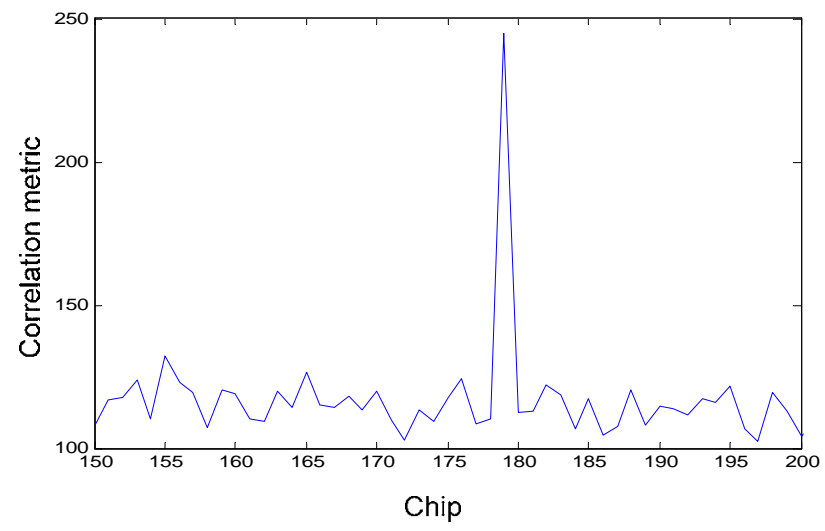

Fig. 8: Timing metric in modified algorithm

The modified algorithm shows good performance in AWGN with well-defined timing metric peak, but it is not the case in fading channels. Same as in other algorithms mentioned above, the main reason is that the received power is concentrated not only in the direct ray but also in other reflected rays with different delays. As result, the maximum timing peak corresponds to the position that is delayed several samples comparing to exact timing.

In case of multi-path fading channel, there are several peaks occurring in the timing metric window, corresponding to the delayed rays. The peak corresponding to correct timing must be the one that has much differences compared to the remaining ones. Therefore, to define the exact timing, we propose a different coefficient $\alpha$. Then, the timing is found by searching the peak that is greater than $\alpha$ time of the previous maximum value ( $\alpha$ is some preset value at the receiver).

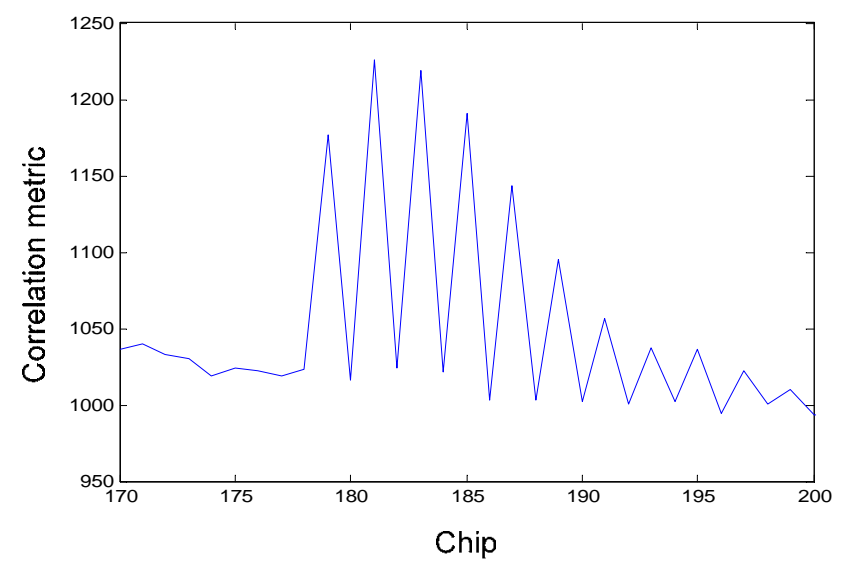

Fig. 9: Several peaks occur in fading channels 


\section{SIMULATION RESULTS}

In this section, we present the simulation results to confirm the performance of the modified algorithm. The MC-CDMA system has number of sub-carriers of $N_{c}=512$ with the frame length of 7, the frames used for averaging are 3, spreading factor is $S F=32$, guard interval is 128 chip, modulation method is QPSK, spreading code is Walsh-Hadamard, the bandwidth is $80 \mathrm{MHz}$ with the channel spacing between sub-carries of $156.25 \mathrm{Khz}$, and the Doppler frequency is $80 \mathrm{~Hz}$. The fading channel is exponential decay with number of paths of 24 , SNR of $6 \mathrm{~dB}$.

\section{CONCLUSION}

In this paper, we presented some timing synchronization algorithms proposed for the MCCDMA systems using time-multiplexed and frequency-multiplexed synchronization channels, and proposed one modified algorithm to improve the performance of the whole system. The modified algorithm uses the different coefficient to find the peak of correlation metric with high correction. The simulation results show that the timing detection probability is much higher compared to previous algorithms.

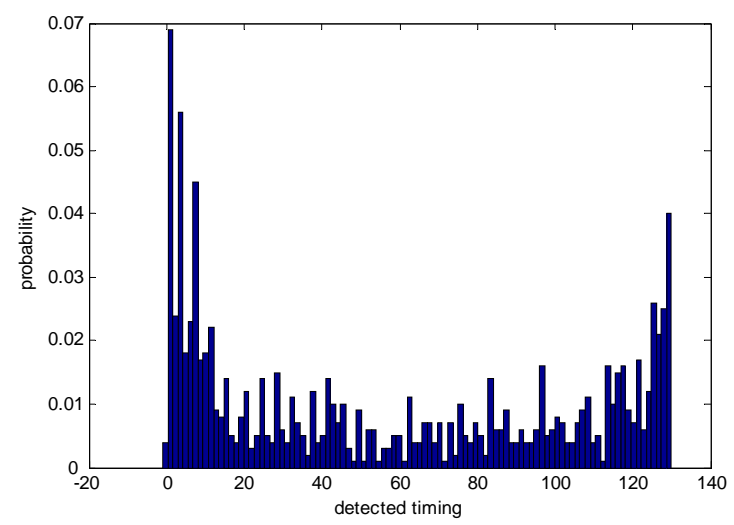

Fig. 10: Timing detection probability in original algorithm

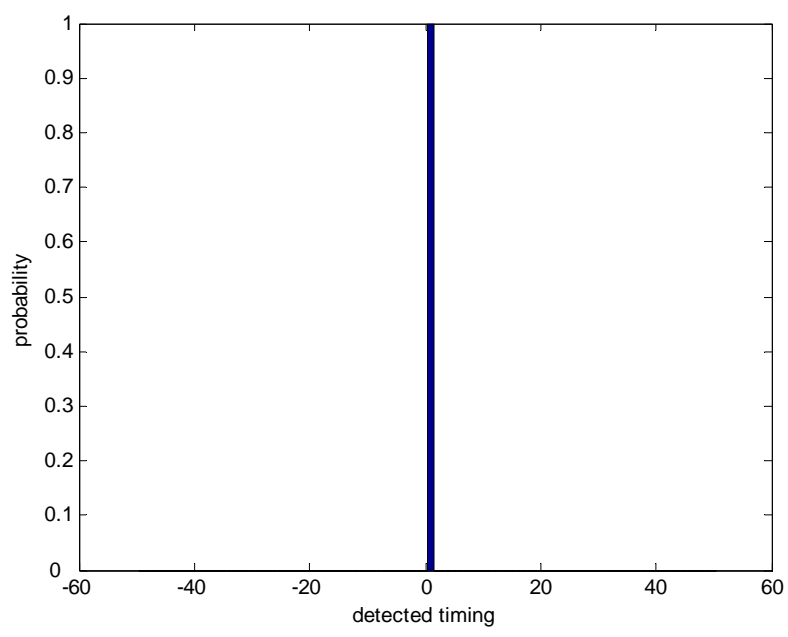

Fig. 11: Timing detection probability in modified algorithm 


\section{REFERENCES}

1. Hara, S. and Prasad, R. (1999), Design and Performance of Multicarrier CDMA System in Frequency-Selective Rayleigh Fading Channels, IEEE Trans. Veh. Technol., vol. 48(5), pp.1584-1595.

2. Nagate, A., Masui, H., and Fujii, T. (2003), A Study on Channel Estimation Methods for MC-CDMA Systems, in Proc. VTC'03 spring, pp. 2101-2105.

3. Matsumoto, A., Miyoshi, K., Uesugi, M., and Kato, O. (2002), A Study on Time Domain Spreading for OFCDM,' in Proc. WPMC'02, pp. 725-728.

4. Yukiko Ishii, Kenichi Higuchi, and Mamoru Sawahashi (2002), Three-Step Cell Search Algorithm Employing Synchronization and Common Pilot Channels for OFCDM Broadband Wireless Access, IEICE Trans. Commun., vol. E85-B.

5. Schmidl, M. and Cox, C. (1997), Robust frequency and timing synchronization for OFDM, IEEE Transactions on Communications, vol. 45, pp.1613-1621.

6. Byungjoon Par, Hyunsoo Cheon, Changeon Kang, and Daesik Hong (2002), A novel timing estimation method for OFDM systems, Global Telecommunications Conference, vol. 1, pp. 269-272. 